Sühl-Strohmenger, Wilfried; Tschander, Ladina (Hrsg.): Praxishandbuch Schreiben in der Hochschulbibliothek: De Gruyter Saur, 2019. ISBN 978-3-11-059116-3. EUR 79,95

Besprochen von Prof. Dr. Ursula Georgy: TH Köln - Technology, Arts, Sciences, Institut für Informationswissenschaft, Gustav-HeinemannUfer 54, D-50968 Köln, E-Mail: ursula.georgy@th-koeln.de

https://doi.org/10.1515/bfp-2020-0012

Das Buch Schreiben in der Hochschulbibliothek ist ein weiteres aus der Serie der Praxishandbücher des De Gruyter Verlages, und - um es vorweg zu nehmen - es wird dem Anspruch der Praxis auch voll gerecht.

\title{
Relevanz des Themas
}

Das Thema Wissenschaftliches Schreiben hat an den Hochschulen in den letzten Jahren eine immer größere Bedeutung erlangt. Und es darf vermutet werden, dass dem Thema in den nächsten Jahren eine noch größere Bedeutung zukommt, da dem traditionellen Schreiben in Schule und Freizeit eine immer geringere Bedeutung zukommt. Festzuhalten bleibt aber, dass schriftliche Kompetenzen auch in Zukunft relevant bleiben werden. Daher ist es unabdingbar, Studierenden diese Kompetenzen zu vermitteln, und zwar idealerweise vom ersten Semester an. Fast alle Hochschulen bieten inzwischen Veranstaltungen zu dem Thema an. Aber wie wird Wissenschaftliches Schreiben am besten vermittelt - und durch wen? Antworten auf diese Fragen liefert dieses Praxishandbuch in zahlreichen Beiträgen, geschrieben von Autoren aus verschiedenen Disziplinen sowie Hochschulen und von Wilfried Sühl-Strohmenger sowie Ladina Tschander hervorragend zusammengetragen.

Im Mittelpunkt der Ausführungen stehen vor allem die praktische Realisierung von Angeboten zum Wissenschaftlichen Schreiben sowie persönliche Erfahrungen von Lehrenden - insbesondere aus der Sicht von Hochschulbibliotheken. Diese haben sich in den letzten Jahren dieses Themas verstärkt angenommen. Wissenschaftliches Schreiben zeichnet sich durch Formalia und Fachspezifika aus. Und die Schnittstelle zwischen beiden Anforderungen können Hochschulbibliotheken schon allein durch ihre Personalstruktur, z. B. in den Fachreferaten, gut bedienen. Hinzu kommt, dass im Allgemeinen nur die Bibliothek einer Hochschule die zum Schreiben notwendige Infrastruktur sowie die Dienstleistungen wie Vermittlung von Informationskompetenz etc. liefern kann. Und damit ist dieses Buch auch eine Leistungsschau der Hochschulbi- 
bliotheken in einem Bereich, den vor einigen Jahren kaum jemand ihnen zugeordnet hätte; das Thema wurde fast ausschließlich von den Kompetenzzentren und/oder Schreibzentren reklamiert.

\section{Beiträge}

Die beiden Herausgeber Wilfried Sühl-Strohmenger und Ladina Tschander haben die insgesamt 24 Beiträge ihres Handbuches drei Themenbereichen zugeordnet:

I. Grundlagen und Rahmenbedingungen,

II. Angebote und Aktivitäten sowie

III. Kooperationen und Netzwerke.

Jeder Teil beginnt mit einer Einleitung der Herausgeber, sodass die Leser einen guten Überblick zu der Schwerpunktsetzung und eine thematische Einordnung der einzelnen Beiträge erhalten. Der erste Themenblock stellt vor allem theoretische und (schreib)didaktische Grundlagen in den Vordergrund und schlägt den Bogen bis hin zu Open Science und Open Access. In den Beiträgen der Themenblöcke II und III werden vor allem konkrete Umsetzungen für Angebote des Wissenschaftlichen Schreibens beschrieben. Die Texte sind im Allgemeinen so verfasst, dass sie für andere Bibliotheken bzw. Kompetenzzentren und Schreibwerkstätten in den Hochschulen hilfreiche Hinweise geben. Diese Ausführungen beziehen sich zum einen auf die organisatorische Ebene, z.B. die Zusammenarbeit zwischen Bibliothek und Fakultäten bzw. Studiengängen sowie zwischen Bibliothek und Kompetenzzentrum/ Schreibwerkstatt, zum anderen auf die Ausgestaltung konkreter Angebote/Veranstaltungen.

Es wurden exemplarisch nur jeweils ein Beitrag aus den drei Teilen herausgegriffen, die aber trotzdem bereits die Breite und gleichzeitig die Tiefe der Texte demonstrieren: Gabriele Fahrenkrog, Rudolf Mumenthaler und Karsten Schuldt haben ihren Beitrag, der den „Grundlagen und Rahmenbedingungen“ zugeordnet ist, mit „Schreiben im digitalen Raum“ überschrieben. Besonders gut stellen die Autoren heraus, dass die Zukunft des Schreibens vor allem zukünftig zunehmend digital erfolgen wird. Dies setzt aber voraus, dass auch die Bibliotheksmitarbeiter bzw. Lehrenden selbst über digitale Medien-, Informations- und Publikationskompetenz verfügen müssen. In ihrem Beitrag gehen die drei Autoren ausführlich auf den Einsatz verschiedener Tools ein. Und einen breiten Raum nimmt das Thema Open Educational Resources (OER) ein, da aus der Sicht der Autoren OER die Prozesse des Schreibens verändert, „denn dem Konzept von OER ist das Verändern, Bearbeiten und Remixen inhärent“ (S. 94). Ungewöhnlich ist das Fazit des Beitrags: Eine Online-Diskussion der Autorin und der Autoren - eine möglicherweise ebenfalls zukunftsweisende Form des Schreibens bzw. des Verschriftlichens. Ingrid Sand und Andrea Thiel (Teil II) bezeichnen ihren Beitrag als Praxisbericht, der den Prozess eines schreibdidaktischen Angebotes in der Hochschulbibliothek der TH Mittelhessen beschreibt. Die Autorinnen sehen dabei die Informationskompetenz als notwendige Teilkompetenz des Schreibens. Sehr detailliert werden personelle, organisatorische und technische Infrastruktur sowie das Angebot selbst beschrieben. Carina Gröner und Edeltraud Haas (Teil III) gehen in ihrem Beitrag auf Kollaborationen an der Universität St. Gallen ein. Im Mittelpunkt steht eine offene Schreibberatung - in Deutsch und Englisch - in der Bibliothek, bei der es sich um ein gemeinsamer Service von Bibliothek und Writing Lab handelt, der ohne Voranmeldung der Studierenden in Anspruch genommen werden kann und durchgängig das ganze Jahr angeboten wird. Sehr ausführlich gehen die Autorinnen auf die Qualifikation der Schreibberater, die Art der Anfragen, den Informationsbedarf und die Nachfrage ein.

Diese exemplarisch herausgegriffenen Beispiele aus den drei Themenschwerpunkten zeigen, dass die Angebote von Bibliotheken zum Wissenschaftlichen Schreiben sehr vielfältig sind und sehr unterschiedliche Schwerpunkte setzen. Und eines haben alle Beiträge des Praxishandbuchs gemeinsam: die sehr anschauliche, praxisund vor allem transferorientierte Darstellung. Die Herausgeber haben eine sehr gute Auswahl bzgl. der Autoren und Themen getroffen. Sehr positiv muss herausgestellt werden, dass das Praxishandbuch deutlich mehr ist als eine Ansammlung von Beispielen; sie sind systematisch geordnet und weisen wenig Redundanzen auf. So machen die Beiträge bereits beim Lesen Lust, sich des Themas anzunehmen. Die teils sehr konkreten Anregungen, die in dem Buch gegeben werden, müssten weitere Hochschulbibliotheken ermutigen, die beschriebenen Ansätze, Methoden und Vorgehensweisen auf Relevanz für die eigene Bibliothek zu prüfen, auf die eigene Einrichtung zu adaptieren und in das eigene Angebotsportfolio zu integrieren sowie zu erproben. Aus Sicht der Rezensentin kommt vor allem Teil III (Kooperationen und Netzwerke) eine besondere Relevanz zu. Die Zeiten, in denen (zentrale) Einrichtungen einer Hochschule, ohne zu kooperieren oder sich zu vernetzen, existieren können, sind endgültig passé. Kooperationen und Netzwerke sind für Hochschulbibliotheken die große Chance, sich in diese mit ihren Stärken und Alleinstellungsmerkmalen einzubringen, was in den Beiträgen des dritten Teils auch durchgängig betont wird. Und genau damit wirken und bleiben Bibliotheken glaubwürdig, 


\section{Formalia}

Das Buch entspricht dem hohen Standard des De Gruyter Verlags und ist gut lesbar. Allerdings wäre es sehr wünschenswert, wenn der Verlag Fotos, Graphiken durchgängig farbig drucken würde, vor allem wenn ein Handbuch wie dieses, in Summe sehr wenige Fotos und Grafiken enthält. Farbdruck ist vielfach kein „nice to have“ sondern ein „must have“. Beispiel: Die sehr interessante Abbildung zur „Nutzungsfrequenz der Rechercheberatung nach Zeitpunkt im Semester und nach Studienstufe der NutzerInnen" an der Universität St. Gallen ist leider fast wertlos, da die Legende(n) den Grafiken aufgrund des fehlenden Kontrastes der Grautöne nicht zuzuordnen sind (S. 269).

Eine Besonderheit weist das Handbuch auf, und zwar hinsichtlich des Literaturverzeichnisses. Es gibt ein gemeinsames Verzeichnis für alle Beiträge am Ende des Buches - ungewöhnlich für ein Herausgeberwerk. Und dieses zentrale Literaturverzeichnis darf und muss kritisch diskutiert werden: Wenn z.B. einzelne Beiträge elektronisch heruntergeladen werden, enthält der einzelne Beitrag kein Literaturverzeichnis. Zwar lassen sich die Quellen auch aus den einzelnen Kapiteln ermitteln, doch gestaltet sich dies als umständlich, da, wenn eine Quelle in einem Beitrag mehrfach zitiert wird, nur ein Verweis auf die jeweilige erste Anmerkung/Fußnote zu der Quelle gegeben wird (z.B. Anm. 7), sodass in dem Beitrag erst geblättert/gescrollt werden muss, um an die vollständige Literaturquelle $\mathrm{zu}$ gelangen. Und auch wenn man das Literaturverzeichnis mit herunterlädt, hat man keinen Überblick, welche Literatur in dem Beitrag zitiert wurde. Einen tatsächlichen Mehrwert würde ein gemeinsames Literaturverzeichnis nur dann liefern, wenn zu der jeweiligen Quelle angegeben worden wäre, in welchen Beiträgen die Quelle genannt wurde. Dann wäre aus dem Literaturverzeichnis auch zu entnehmen, welche Quellen in mehreren Beiträgen zitiert werden. Damit würde den Quellen eine Art „Impact-Faktor“ zugeordnet.

\section{Fazit und Ausblick}

Das Buch beschränkt sich auf das Wissenschaftliche Schreiben im Studium (Bachelor, Master), wobei es wünschenswert gewesen wäre, zu Beginn der einzelnen Beiträge deutlicher darzulegen, welches Level des Wissenschaftlichen Schreibens diskutiert wird. So macht es einen Unterschied, ob es um das Schreiben der ersten Hausarbeit im ersten Semester eines Bachelor-Studiengangs oder das Verfassen einer Master-Thesis geht. Nun gibt es ja noch ein Wissenschaftliches Schreiben jenseits von Bachelor und
Master - das Wissenschaftliche Publizieren. Daher sei an dieser Stelle die Frage erlaubt: Wann folgt ein Handbuch zum Wissenschaftlichen Publizieren? Das Wissenschaftliche Publizieren hat in den letzten Jahren einen solch grundlegenden Wandel durchgemacht, sodass es ein mindestens so dringliches Thema darstellt. Und nach diesem hervorragenden Buch würde man sich von den beiden Herausgebern ein „Praxishandbuch Publizieren in der Hochschulbibliothek" wünschen.

\section{Zielpublikum}

Bibliotheken oder/und Bibliotheksabteilungen, Kompetenzwerkstätten sowie Akademische Schreibzentren von Hochschulen mit ihren Mitarbeitern, Dozenten, aber auch Studierende, die sich mit dem Thema des wissenschaftlichen Schreibens intensiv(er) auseinandersetzen möchten bzw. müssen. 